This document is the accepted manuscript version of the following article:

Ih7i, J., Green, D. C., Lynch, C., Holden, M. A., Lee, P. A., Zhang, S., ... Meldrum, F. C. (2019). Super-resolution microscopy reveals shape and distribution of dislocations in single-crystal nanocomposites. Angewandte Chemie International Edition, 58(48), 17328-17334. https://doi.org/10.1002/anie.201905293

\title{
Super-Resolution Microscopy Reveals Shape and Distribution of Dislocations in Single Crystal Nanocomposites
}

Johannes Ihli*, David C. Green, Christophe Lynch, Mark A. Holden, Phillip A. Lee, Shuheng Zhang, Ian K. Robinson, Stephen E. D. Webb and Fiona C. Meldrum*

Dr J. Ihli

Paul Scherrer Institut, 5232 Villigen PSI, CH

Dr J. Ihli, Dr D.C. Green, Dr M.A. Holden, Mr P.A. Lee, Dr S. Zhang, Prof F.C. Meldrum School of Chemistry, University of Leeds, Leeds, LS2 9JT, UK.

Dr C. Lynch, Dr SED Webb

Central Laser Facility, Science and Technology Facilities Council, Research Complex at Harwell, Rutherford Appleton Laboratory, Didcot OX11 0QX, UK.

Dr M.A. Holden

School of Physical Sciences and Computing, University of Central Lancashire, Preston, PR1 2HE, UK

Prof IK Robinson

London Centre for Nanotechnology, University College London, London, WC1H 0AH, UK Brookhaven National Lab, Upton NY 11973, USA

E-mail: Johannes.Ihli@psi.ch and F.Meldrum@leeds.ac.uk

Keywords: calcium carbonate, crystal growth, defect, dislocation, nanostructure, STORM

With their potential to offer new properties, single crystals containing nanoparticles provide an attractive class of nanocomposite materials. However, to fully profit from these, it is essential that we can characterise their 3D structures, identifying the locations of individual nanoparticles, and the defects present within the host crystals. Using calcite crystals containing quantum dots as a model system, we here use 3D stochastic optical reconstruction microscopy (STORM) to locate the positions of the nanoparticles within the host crystal. The nanoparticles are shown to preferentially associate with dislocations in a manner previously recognised for atomic impurities, rendering these defects visible by STORM. Our images also demonstrate that the types of dislocations formed at the crystal/ substrate interface vary according to the nucleation 
face, and dislocation loops are observed that have entirely different geometries to classic misfit dislocations. This approach offers a rapid, easily accessed, and non-destructive method for visualising the dislocations present within crystals, and gives insight into the mechanisms by which additives become occluded within crystals.

\section{Introduction}

Doping crystals with foreign species is a powerful strategy for tuning properties and creating new functional materials, where judicious combination of the host and additive can result in new properties including colour, magnetism and conductivity. ${ }^{[1]}$ Traditional solid solutions are formed by substituting atoms/ ions in the host crystal for others of comparable size and charge, while many crystals can be doped with atomic or molecular species that reside in interstitial sites in the lattice. It is now also recognised that particulate species including organic and inorganic nanoparticles, ${ }^{[2]}$ protein aggregates, ${ }^{[3]}$ and even liquids ${ }^{[4]}$ can be incorporated within single crystals, which vastly increases the range of potential material combinations. However, the degree of loading of the particles varies enormously, where levels of only $0.01 \mathrm{wt} \%$ were achieved when simple salts such as $\mathrm{NaCl}$ were precipitated in the presence of inorganic nanoparticles $^{[5]}$ as compared with over $20 \mathrm{wt} \%$ in $\mathrm{ZnO}$ and calcite crystals for nanoparticles functionalised with coronas of polymer chains. ${ }^{[2 a, 6]}$

Looking beyond composition, occlusions can also give rise to enhanced materials properties due to their influence on the crystal lattice itself. For example, compressive stresses associated with Mg-rich nanoparticle occlusions in calcite give rise to increased hardness, ${ }^{[7]}$ while amino acid occlusions in $\mathrm{ZnO}$ result in a shift in the band gap. ${ }^{[8]}$ The introduction of occlusions into a crystal can also generate defects, whose character, density and mobility is fundamental to 
many important properties including ionic conductivity, mechanical properties, and crystal growth and dissolution. ${ }^{[9]}$ Determination of the mechanisms underlying particle incorporation within crystals, and the relationship between defect structures and occlusion, therefore promises the ability to control and fully profit from this synthetic strategy.

Here, we demonstrate the use of 3D stochastic optical reconstruction microscopy (STORM), a super-resolution fluorescence-based microscopy technique, ${ }^{[10]}$ to characterise the internal structures of nanocomposite crystals. Studying single crystals of calcite $\left(\mathrm{CaCO}_{3}\right)$ containing fluorescent nanoparticles, we show that at low occlusion levels the nanoparticles preferentially associate with, and form an atmosphere around dislocations within the crystals. This offers new insight into the mechanism of interaction, and occlusion of particulate additives within these crystals. STORM therefore provides a rapid, non-destructive and readily accessed method for visualising the locations and shapes of dislocations in $3 \mathrm{D}$, and correlating these with the morphology of the crystal.

\section{Results}

STORM generates images based on single molecule/ particle detection. ${ }^{[10]}$ Fluorophores that can be optically switched between active and deactivated states are employed, where the stochastic nature of the emission is such that only a fraction of the fluorophores emit when an individual frame is recorded. The shape of the emission around each centre is described by an imaging-system-defined point-spread function (PSF), where the introduction of astigmatism or defocusing into the system enables the positions of individual emitters to be localised in three dimensions with nanometre precision. By recording multiple images the positions of a population of distinct fluorophores can be identified, and an overall image of the sample can be reconstructed. 
While the original fluorescent label for STORM consisted of an activator and reporter dye pair, in which the reporter dye switches between fluorescent -on and dark -off states, advances in methods such as direct STORM, ${ }^{[11]}$ have eliminated the need for access to a large reservoir of the activator during image acquisition. Yet, methods using fluorophores commonly still require access to a solution environment or an oxygen scavenger to function optimally.

Given our interest in the incorporation of particulate additives (that occupy isolated environments inside the crystal), we employed $6 \mathrm{~nm}$ carboxylic acid functionalised CdSeS/ZnS quantum dots (QDs) as fluorescent labels. These QDs exhibit a stochastic, on-off "blinking" behaviour that is independent of solution or oxygen access. ${ }^{[12]}$ The carboxylic acid surface functionalisation of the QDs was chosen to ensure solubility in the crystal growth solution and to promote interaction with the surfaces of the growing crystals. Calcite $\left(\mathrm{CaCO}_{3}\right)$ was selected as the host crystal as there is considerable precedent for its ability to occlude foreign species, and its crystal growth mechanisms have been well characterised.

Calcite single crystals occluding QDs were precipitated on glass cover slips by exposing solutions of $2.5 \mathrm{mM}$ or $10 \mathrm{mM}$ calcium chloride containing QDs to ammonium carbonate $\operatorname{vapour}^{[13]}$ (Figure 1a). A fluorescent dye, 3-hydroxynaphthalene-2,7-disulphonic acid (HNDS) was also added to the growth solution. While the QDs only occupy specific locations within each calcite crystal, the dye is distributed uniformly. ${ }^{[14]}$ Addition of the dye therefore enabled the morphologies of the crystals to be determined using 3D widefield fluorescence microscopy (WFM), such that the locations of the QDs identified using STORM could be correlated with the crystal structure. 
Scanning electron microscopy (SEM) of the product crystals showed that they were 3-20 $\mu \mathrm{m}$ in size, had rhombohedral morphologies, and were randomly oriented with respect to the glass substrate (Figure 1b). Raman microscopy and infra-red spectroscopy confirmed that they were calcite (Figure S1), while thermogravimetric analysis (TGA) demonstrated low levels of occluded QDs (<1wt\%) (Figure S2). Initial studies focused on nanocomposite crystals with $\{104\}$ basal planes where 3D STORM and WFM datasets were recorded sequentially. The STORM localisation micrographs were acquired from a $20 \times 20 \times 3 \mu \mathrm{m}$ field of view with average localisation precisions of $\approx 27.8 \mathrm{~nm}( \pm 2.9 \mathrm{~nm})$ in the $\mathrm{x}-\mathrm{y}$ plane and $38.7 \mathrm{~nm}( \pm 18.9 \mathrm{~nm})$ in the $\mathrm{z}$ direction.

Figure 2 displays volume renderings of two calcite crystals precipitated from $2.5 \mathrm{~mm}$ (Figure 2a) and $10 \mathrm{~mm}$ (Figure 2b) calcium chloride solutions. WFM was used to generate images of the crystals as shown in semi-transparent grey, with a spatial-resolution of $\approx 300 \mathrm{~nm}$ in the $\mathrm{x}-\mathrm{y}$ plane. The locations of the QDs, in turn, were derived from STORM datasets. The QD localisations are mapped using a divergent colour scale that scales with the number of detected photons per QD localisation. The corresponding intensity and localisation histograms are presented in Figures $2 \mathrm{c}$ and $2 \mathrm{~d}$. These provide an estimate on the relative number of occluded QDs across samples based on the detected number of localisations, where the number of photons detected also has some dependence on the sample depth. Higher QD occlusion and clustering occurs in the crystals grown from the more supersaturated solution. The 3D STORM data are acquired from a limited depth range of $\approx 2.8 \mu \mathrm{m}$ such that only a section of the calcite nanocomposite crystal was sampled. Data were collected adjacent to the glass substrate throughout (Figure 1a). This limited height is due to the spatial reach of the helical PSF used. Increased sample heights can be imaged by the acquisition of multiple depth-interleaved STORM datasets. 
The images clearly show that the QDs are occluded at low levels within the crystal host, but that they are concentrated in a number of discrete domains. These take the form of lowcurvature arcs that originate from the nucleating crystal face and which are directed roughly perpendicular to the interface (Movie S1). The locations and shapes of these structures are consistent with the dislocations that are commonly found in calcite. ${ }^{[15]}$ Studies of dislocations in unstrained calcite using etching methods and X-ray topography have suggested dislocation densities in the order of $\rho=10^{3} \mathrm{~cm}^{-2} \cdot{ }^{[15 \mathrm{~b}]}$ The crystals shown in Figure 2, in contrast, contain roughly 10 identifiable dislocations, and thus dislocation densities in the order of $3 \times 10^{7} \mathrm{~cm}^{-2}$. This is indicative of the crystal experiencing strain, where comparable densities have been measured for calcite single crystals that have undergone small uniaxial compressions. ${ }^{[15 b, 16]}$

The influence of the crystal orientation on the dislocations present was then investigated by characterising calcite nanocomposite crystals with $\{012\}$ basal planes. STORM image reconstructions of occluded QDs in two crystals in this orientation are shown in Figures 3a and 3b. Again, the QDs are sparsely distributed throughout these crystals, but are concentrated in discrete domains. While some of these features exhibit similar geometries to the dislocations in the $\{104\}$ oriented crystals, running approximately perpendicular to the basal plane, clusters roughly in the centre of the STORM reconstructions or the crystal also take the form of dislocation loops. These are magnified in Figures $3 \mathrm{a}$ and $3 \mathrm{~b}$ and Movie S2 and are oriented such that ends of the loop terminate at the crystal/ substrate interface, and the loop themselves lie parallel to $\{104\}$ faces. The preferential calcite slip system comprises $\{104\}$ and $\{012\}$ planes and $<\overline{4} \overline{2} 1>$ slip directions, such that dislocation loops must lie in one of these planes. ${ }^{[17]}$

Further confirmation of these structures as dislocations is provided by our recent study of $\{012\}$ oriented calcite crystals located on carboxyl-terminated self-assembled monolayers (SAMs) 
using Bragg coherent diffraction imaging (BCDI). ${ }^{[18]}$ This synchrotron-based technique enables the visualisation of dislocations in 3D. BCDI of $2 \mu \mathrm{m}$ calcite crystals (ie at an earlier stage of development) precipitated in the absence of QDs revealed the presence of single dislocation loops within each crystal (Figure 3c) where these were of comparable sizes and orientations to those seen here by STORM. ${ }^{[18]}$ Notably, these loops exhibit entirely different geometries to the traditional misfit dislocations that form between two crystalline materials with a close lattice match. ${ }^{[19]}$ These generally nucleate at the free surface of the growing crystal, and the loop then grows until its apex reaches the crystal/ substrate interface. That these unusual dislocation loops have now been observed in calcite crystals nucleated on different substrates (glass, and SAM/ gold/ glass) and using independent techniques further validates their existence and emphasises the role of strain present at the crystal/substrate interface in generating these structures.

\section{Discussion}

The ability to image dislocations within crystalline materials, and to monitor how they move and interact under external stimuli is critical to understanding many materials properties. While it has long been possible to image individual dislocations using transmission electron microscopy $(\mathrm{TEM}),{ }^{[20]}$ visualising these defects in three dimensions has proven far more challenging. Electron tomography now allows 3D images of defects such as stacking faults, grain boundaries and dislocations to be generated with atomic resolution. ${ }^{[21]}$ However, this can only be achieved for nano-scale crystals, or sections prepared from larger samples, and measurements are commonly made in vacuum. A range of X-ray based methods have also been widely used, ${ }^{[22]}$ perhaps most notably BCDI, which enables a dislocation and its associated strain field to be visualised down to $10 \mathrm{~nm}$ in spatial resolution. ${ }^{[9 \mathrm{~b}, 15 \mathrm{a}]}$ The disadvantages of 
this technique include the requirement for a synchrotron source, the complexity of the data analysis and there are again restrictions on the sample size and level of strain.

Use of a STORM-based approach to visualise the principal crystallographic defects in 3D therefore provides a promising extension to these existing X-ray and electron-based methodologies. While we cannot entirely exclude the possibility that the nanoparticles could contribute to the formation of dislocations, the fact that the number density the shapes of the dislocations is comparable to crystals formed in their absence, ${ }^{[18]}$ suggest that this is unlikely. In situ AFM analysis of calcite growth in the presence of nanoparticles has also shown that propagation of the surface steps past adsorbed particles is facile. ${ }^{[23]}$ The success of the method is principally based on three factors: the unitisation of QDs, such that signal is acquired from truly isolated environments, ${ }^{[24]}$ the suppression of background signals compared to widefield or confocal laser scanning fluorescence microscopy (CLSFM), and the extension to threedimensions. Indeed, visualisation of the nanocomposite crystals using (CLSFM) failed to resolve the dislocations present, demonstrating that the same information could not be retrieved by simpler means (Figure S3).

Our observation that QDs preferentially associate with dislocations is itself also fascinating. That atmospheres of impurity atoms form around dislocations is well-recognised in the socalled Cottrell Effect. ${ }^{[25]}$ We are not aware of any reports of this effect with molecules or nanoparticles, but it is our expectation that it may actually be quite widespread, and has been overlooked due to the absence of techniques that can visualise both the dislocations and occluded material. The Cottrell Effect is considered to arise due to a reduction in the strain fields arising from the dislocation and impurities thanks to their association. ${ }^{[25-26]}$ This may also occur during nanoparticle occlusion, where incorporation of any nanoparticle within calcite 
gives rise to local strain fields, as revealed by high resolution powder XRD studies of single crystal nanocomposites. ${ }^{[2 a, 4,7]}$

Kinetics also play a significant role in the occlusion of additives within crystals, however, where superior occlusion of additives that bind weakly to a crystal is expected during rapid as compared with slow growth. ${ }^{[27]}$ The association of additives with a crystal surface is a dynamic process, where occlusion depends on the balance between the rates of additive adsorption, additive desorption, and step propagation. Occlusion occurs if a nanoparticle remains bound to a step edge long enough for it to be entrapped by the propagation of neighbouring step edges. ${ }^{[23]}$ Under the experimental conditions used here - where nanoparticle occlusion occurs at very low levels - the strain field associated with the dislocation core may be sufficient to reduce the nanoparticle desorption rate such that some nanoparticle occlusion can occur.

This study also further demonstrates the potential of super-resolution microscopy in materials science. While principally used to investigate biological specimens, super-resolution microscopy is attracting increasing attention as a method of characterising synthetic materials ranging from polymers, to carbon nanostructures, catalysts and inorganic systems. ${ }^{[28]}$ As prominent examples of its application to inorganic materials, super-resolution microscopy has been used to visualise the active sites on catalytic nanoparticles, ${ }^{[29]}$ and to study transport processes in porous materials. ${ }^{[24 a, 24 b]}$ A number of recent papers have also used it to characterise perovskite materials, imaging traps, ${ }^{[30]}$ degradation processes ${ }^{[31]}$ and carrier diffusion. ${ }^{[32]}$ Of particular relevance to the current work, STORM has been used to visualise labelled gelatine fibres, ${ }^{[24 c]}$ and proteins, ${ }^{[24 d]}$ within $\mathrm{CaCO}_{3}$ crystals. However, few of these studies report 3D images or target the internal structure of the crystalline material.

\section{Conclusions}


We have here presented a novel strategy for visualising extended defects within crystalline materials in 3D, where this relies on (i) occluding low concentrations of fluorescent nanoparticles within the crystals, where these principally locate in the vicinity of dislocations and (ii) using 3D super-resolution microscopy to map the locations of the nanoparticles. This approach is rapid and non-destructive, can be used to examine large samples, operates under ambient conditions and uses instrumentation that is widely accessible. It could also be employed to conduct in situ experiments such as visualising the process of additive incorporation, studying dislocation birth and movement during crystal growth, or amorphous to crystalline phase transformations. Our results provide an independent verification of the formation of dislocation loops with unusual geometries on the nucleation of calcite crystals at substrates, and give further insight into the mechanisms by which additives become occluded within crystals. An improved understanding of this effect will facilitate the design of nanocomposite materials with desired structure/property relationships.

\section{Supporting Information}

Supporting Information is available from the Wiley Online Library or from the author.

\section{Acknowledgements}

This work was supported by an Engineering and Physical Sciences Research Council (EPSRC) Leadership Fellowship (F.C.M and J.I. EP/H005374/1), an EPSRC Platform Grant (F.C.M., S.Z. and M.A.H., EP/N002423/1) an EPSRC Research Grant (F.C.M. and D.C.G., EP/P005233/1) and by the Swiss National Science Foundation (SNF) (J.I. grant number 153556 and 179886). C.L. and I.K.R. were supported by a BBSRC Diamond Fellowship. Localization microscopy experiments were performed using the Octopus Imaging Cluster Facility, which is 
part of the Central Laser Facility at Harwell. Work at Brookhaven National Laboratory was supported by the U.S. Department of Energy, Office of Science, Office of Basic Energy Sciences, under Contract No. DE-SC0012704. 


\section{References}

[1] C.-W. Nan, Q. Jia, MRS Bull. 2015, 40, 719-723.

[2] (a) Y. Y. Kim, K. Ganesan, P. C. Yang, A. N. Kulak, S. Borukhin, S. Pechook, L. Ribeiro, R. Kroger, S. J. Eichhorn, S. P. Armes, B. Pokroy, F. C. Meldrum, Nature Mater. 2011, 10, 890-896; (b) Z. J. Ning, X. W. Gong, R. Comin, G. Walters, F. J. Fan, O. Voznyy, E. Yassitepe, A. Buin, S. Hoogland, E. H. Sargent, Nature 2015, 523, 324-328; (c) Y. J. Liu, H. D. Zang, L. Wang, W. F. Fu, W. T. Yuan, J. K. Wu, X. Y. Jin, J. S. Han, C. F. Wu, Y. Wang, H. L. L. Xing, H. Z. Chen, H. Y. Li, Chem. Mater. 2016, 28, 7537-7543; (d) A. N. Kulak, M. Semsarilar, Y. Y. Kim, J. Ihli, L. A. Fielding, O. Cespedes, S. P. Armes, F. C. Meldrum, Chem. Sci. 2014, 5, 738-743; (e) A. N. Kulak, R. Grimes, Y. Y. Kim, M. Semsarilar, C. Anduix-Canto, O. Cespedes, S. P. Armes, F. C. Meldrum, Chem. Mater. 2016, 28, 7528-7536; (f) D. C. Green, M. A. Holden, M. A. Levenstein, S. Zhang, B. R. G. Johnson, J. Gala de Pablo, A. Ward, S. W. Botchway, F. C. Meldrum, Nat. Commun. 2019, 206; (g) G. Lu, S. Li, Z. Guo, O. K. Farha, B. G. Hauser, X. Qi, Y. Wang, X. Wang, S. Han, X. Liu, J. S. DuChene, H. Zhang, Q. Zhang, X. Chen, J. Ma, S. C. J. Loo, W. D. Wei, Y. Yang, J. T. Hupp, F. Huo, Nature Chem. 2012, 4, 310-316.

[3] (a) H. Y. Li, H. L. Xin, M. E. Kunitake, E. C. Keene, D. A. Muller, L. A. Estroff, Adv. Func. Mater. 2011, 21, 2028-2034; (b) A. Berman, L. Addadi, A. Kvick, L.

Leiserowitz, M. Nelson, S. Weiner, Science 1990, 250, 664-667.

[4] Y. Y. Kim, M. Semsarilar, J. D. Carloni, K. R. Cho, A. N. Kulak, I. Polishchuk, C. T. Hendley, P. J. M. Smeets, L. A. Fielding, B. Pokroy, C. C. Tang, L. A. Estroff, S. P. Baker, S. P. Armes, F. C. Meldrum, Adv. Func. Mater. 2016, 26, 1382-1392.

[5] M. Adam, T. Erdem, G. M. Stachowski, Z. Soran-Erdem, J. F. L. Lox, C. Bauer, J. Poppe, L. V. Demir, N. Gaponik, A. Eychmuller, ACS Appl. Mater. Int. 2015, 7, 23364-23371.

[6] Y. Ning, L. A. Fielding, J. Nutter, A. N. Kulak, F. C. Meldrum, S. P. Armes, Angew. Chem. Int. Ed. 2019, 58, 4302-4307.

[7] I. Polishchuk, A. A. Bracha, L. Bloch, D. Levy, S. Kozachkevich, Y. Etinger-Geller, Y. Kauffmann, M. Burghammer, C. Giacobbe, J. Villanova, G. Hendler, C. Y. Sun, A. J. Giuffre, M. A. Marcus, L. Kundanati, P. Zaslansky, N. M. Pugno, P. Gilbert, A. Katsman, B. Pokroy, Science 2017, 358, 1294-1298. 
[8] M. A. H. Muhammed, M. Lamers, V. Baumann, P. Dey, A. J. Blanch, I. Polishchuk, X. T. Kong, D. Levy, A. S. Urban, A. O. Govorov, B. Pokroy, J. RodriguezFernandez, J. Feldmann, J. Phys. Chem. C 2018, 122, 6348-6356.

[9] (a) A. Ulvestad, A. Singer, J. N. Clark, H. M. Cho, J. W. Kim, R. Harder, J. Maser, Y. S. Meng, O. G. Shpyrko, Science 2015, 348, 1344-1347; (b) J. Ihli, J. N. Clark, N. Kanwal, Y.-Y. Kim, M. A. Holden, R. J. Harder, C. C. Tang, S. E. Ashbrook, I. K. Robinson, F. C. Meldrum, Chem. Sci. 2019, 10, 1176-1185; (c) Y. Y. Kim, J. D. Carloni, B. Demarchi, D. Sparks, D. G. Reid, M. E. Kunitake, C. C. Tang, M. J. Duer, C. L. Freeman, B. Pokroy, K. Penkman, J. H. Harding, L. A. Estroff, S. P. Baker, F. C. Meldrum, Nature Mater 2016, 15, 903-912.

[10] B. Huang, W. Q. Wang, M. Bates, X. W. Zhuang, Science 2008, 319, 810-813.

[11] D. R. Whelan, T. Holm, M. Sauer, T. D. M. Bell, Aust. J. Chem. 2014, 67, 179-183.

[12] V. Rombach-Riegraf, P. Oswald, R. Bienert, J. Petersen, M. P. Domingo, J. Pardo, P. Gräber, E. M. Galvez, Biochem. Biophys. Res. Commun. 2013, 430, 260-264.

[13] J. Ihli, P. Bots, A. N. Kulak, L. G. Benning, F. C. Meldrum, Adv. Func. Mater. 2013, 23, 1965-1973.

[14] D. C. Green, J. Ihli, P. D. Thornton, M. A. Holden, B. Marzec, Y.-Y. Kim, A. N. Kulak, M. A. Levenstein, C. Tang, C. Lynch, S. E. D. Webb, C. J. Tynan, F. C. Meldrum, Nature Commun. 2016, 7, 13524.

[15] (a) J. N. Clark, J. Ihli, A. S. Schenk, Y.-Y. Kim, A. N. Kulak, J. M. Campbell, G. Nisbet, F. C. Meldrum, I. K. Robinson, Nature Mater. 2015, 14, 780-785; (b) I. N. Macinnis, S. L. Brantley, Geochim. Cosmochim. Acta 1992, 56, 1113-1126.

[16] J. H. P. DeBresser, J. Geophys. Res. Solid Earth 1996, 101, 22189-22201.

[17] J. H. P. DeBresser, C. J. Spiers, Tectonophysics 1997, 272, 1-23.

[18] J. Ihli, J. N. Clark, A. S. Cote, Y. Y. Kim, A. S. Schenk, A. N. Kulak, T. P. Comyn, O. Chammas, R. J. Harder, D. M. Duffy, I. K. Robinson, F. C. Meldrum, Nature Commun. 2016, 7, 11878.

[19] (a) R. Hull, J. C. Bean, Crit. Rev. Solid State Mater. Sci. 1992, 17, 507-546; (b) S. C. Jain, A. H. Harker, R. A. Cowley, Phil. Mag. A. 1997, 75, 1461-1515; cJ. H. Van der Merwe, Crit. Rev. Solid State Mater. Sci. 1991, 17, 187-209.

[20] P. B. Hirsch, R. W. Horne, M. J. Whelan, Phil. Mag. 2006, 86, 4553-4572.

[21] (a) C. C. Chen, C. Zhu, E. R. White, C. Y. Chiu, M. C. Scott, B. C. Regan, L. D. Marks, Y. Huang, J. W. Miao, Nature 2013, 496, 74-79; (b) J. Miao, P. Ercius, S. J. L. Billinge, Science 2016, 353, aaf2157. 
[22] R. Tanuma, I. Kamata, J. P. Hadorn, H. Tsuchida, J. Appl. Phys. 2018, 124, 125703.

[23] K. R. Cho, Y. Y. Kim, P. C. Yang, W. Cai, H. H. Pan, A. N. Kulak, J. L. Lau, P. Kulshreshtha, S. P. Armes, F. C. Meldrum, J. J. De Yoreo, Nature Commun. 2016, 7, 10187.

[24] (a) A. Zuerner, J. Kirstein, M. Doeblinger, C. Braeuchle, T. Bein, Nature 2007, 450, 705-708; (b) Z. Ristanovic, A. V. Kubarev, J. Hofkens, M. B. J. Roeffaers, B. M. Weckhuysen, J. Am. Chem. Soc. 2016, 138, 13586-13596; (c) M. F. Fu, A. H. Wang, X. M. Zhang, L. R. Dai, J. B. Li, Angew. Chem. Int. Ed. 2016, 55, 908-911; (d) C. Liu, J. Z. Du, L. P. Xie, R. Q. Zhang, Crys. Growth Des. 2017, 17, 1966-1976.

[25] A. H. Cottrell, B. A. Bilby, Proc. Phys. Soc. Lon. Sec. A 1949, 62, 49-62.

[26] (a) E. Tochigi, Y. Kezuka, A. Nakamura, A. Nakamura, N. Shibata, Y. Ikuhara, Nano Letts. 2017, 17, 2908-2912; (b) S. Duguay, T. Philippe, F. Cristiano, D. Blavette, Appl. Phys. Letts. 2010, 97, 3.

[27] A. A. Chernov, in Springer Series in Solid State Sciences, Vol. 36, Springer-Verlag, 1984.

[28] (a) S. Pujals, N. Feiner-Gracia, P. Delcanale, I. Voets, L. Albertazzi, Nature Rev. Chem. 2019, 3, 68-84; (b) D. Woll, C. Flors, Small Methods 2017, 1, 12.

[29] (a) J. Van Loon, A. V. Kubarev, M. B. J. Roeffaers, Chemnanomat 2018, 4, 6-14; (b) N. M. Andoy, X. Zhou, E. Choudhary, H. Shen, G. Liu, P. Chen, J. Am. Chem. Soc. 2013, 135, 1845-1852; (c) T. Chen, B. Dong, K. C. Chen, F. Zhao, X. D. Cheng, C. B. Ma, S. Lee, P. Zhang, S. H. Kang, J. W. Ha, W. L. Xu, N. Fang, Chem. Revs. 2017, 117, 7510-7537; (d) T. Tachikawa, S. Yamashita, T. Majima, J. Am. Chem. Soc. 2011, 133, 7197-7204.

[30] (a) H. F. Yuan, E. Debroye, E. Bladt, G. Lu, M. Keshavarz, K. P. F. Janssen, M. B. J. Roeffaers, S. Bals, E. H. Sargent, J. Hofkens, Adv. Mater. 2018, 30, 9; (b) A. Merdasa, Y. Tian, R. Camacho, A. Dobrovolsky, E. Debroye, E. L. Unger, J. Hofkens, V. Sundstrom, I. G. Scheblykin, ACS Nano 2017, 11, 5391-5404.

[31] A. Merdasa, M. Bag, Y. Tian, E. Kallman, A. Dobrovolsky, I. G. Scheblykin, J. Phys. Chem. C 2016, 120, 10711-10719.

[32] W. M. Tian, C. Y. Zhao, J. Leng, R. R. Gui, S. G. Jin, J. Am. Chem. Soc. 2015, 137, 12458-12461. 


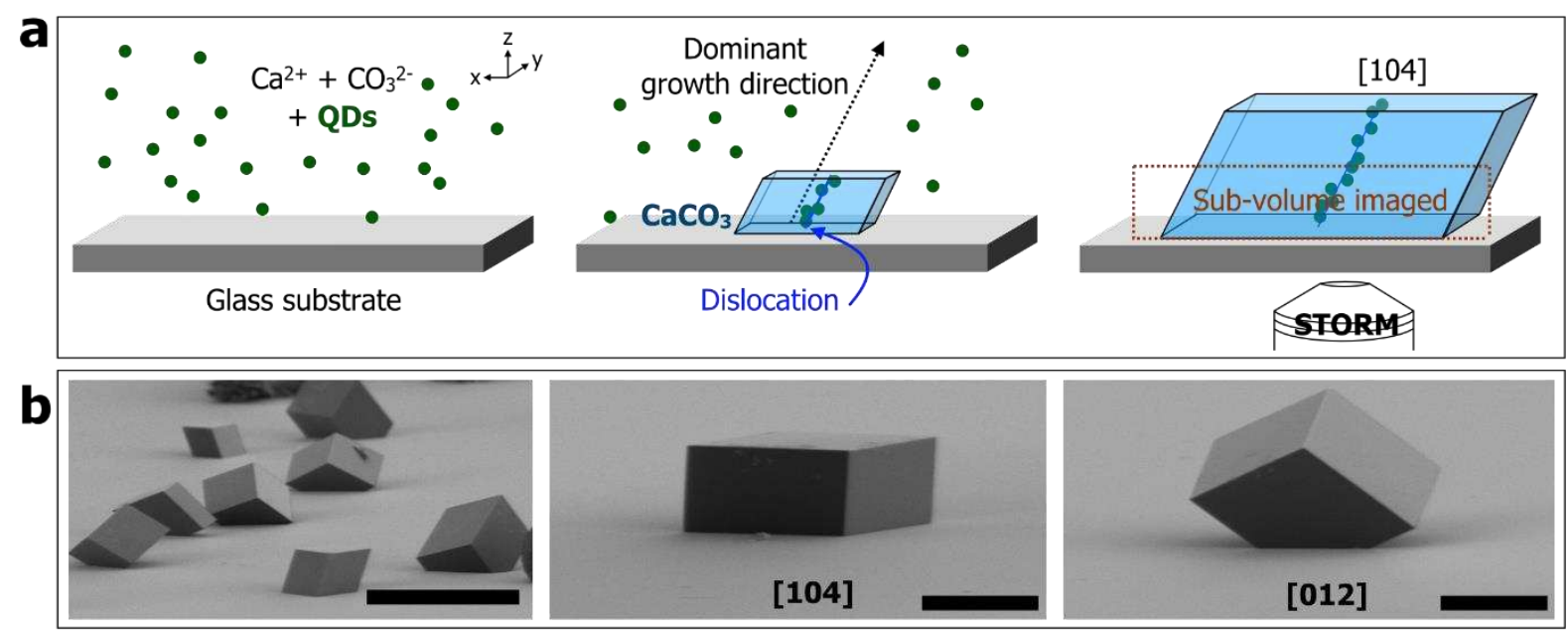

Figure 1. (a) Schematic representation of the de novo precipitation of calcite/quantum dot (QD) nanocomposites. The QD-rich aqueous crystallisation liquor is supersaturated with respect to calcite through the introduction of ammonia and carbon dioxide (i). Nucleation occurs on the glass substrate, followed by growth in a dominant direction, and QDs accumulate at dislocations (ii). Upon completion, the crystals are imaged by STORM within a sub-volume as indicated (iii). (b) Electron micrograph of calcite/QD nanocomposites, where the images show a population of crystals and higher magnification micrographs of crystals with the $\{104\}$ and $\{012\}$ basal planes examined in this study. Scale bars are $10 \mu \mathrm{m}$. 

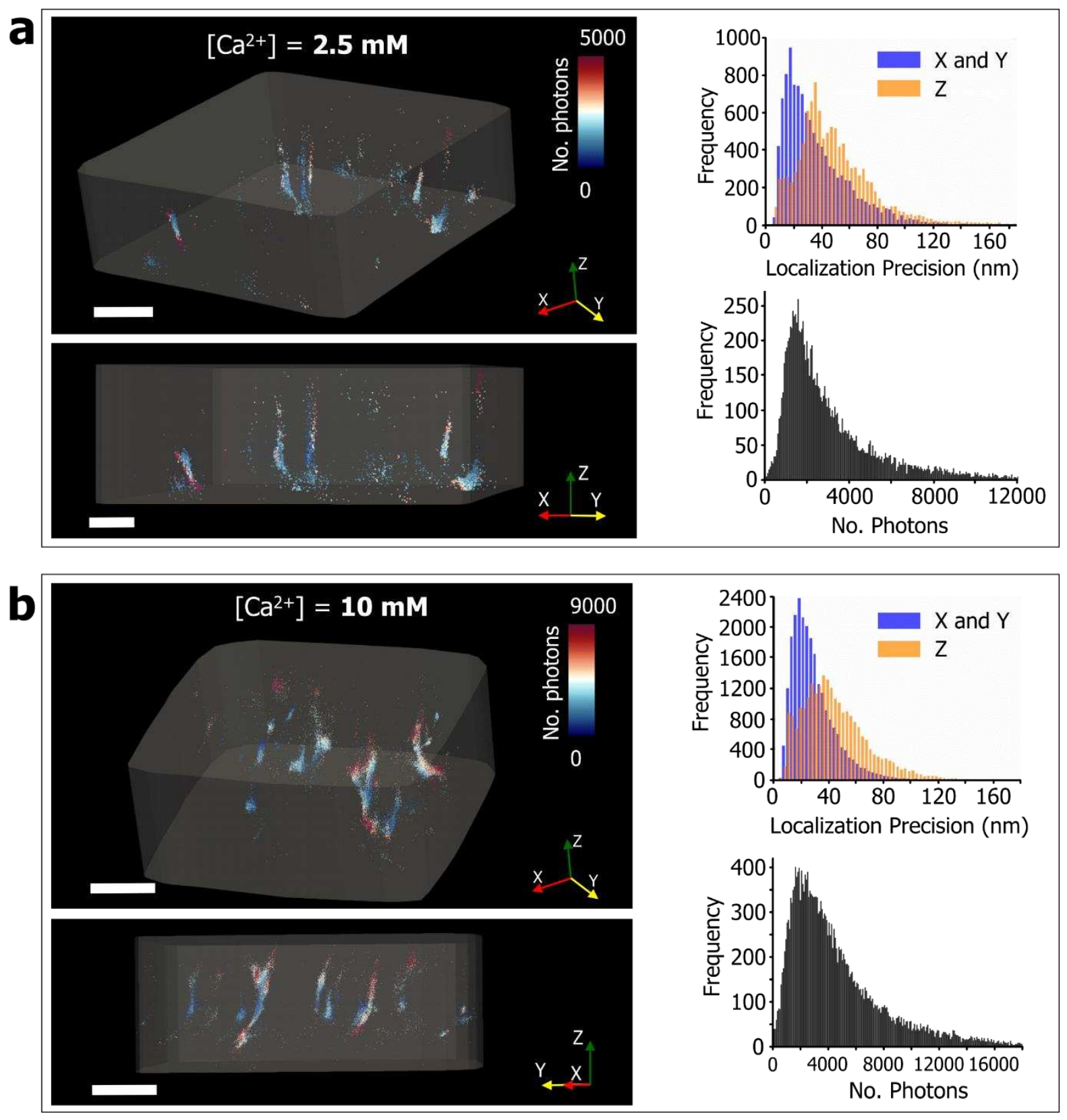

Figure 2. 3D STORM image reconstructions of calcite/quantum dot nanocomposites with $\{104\}$ basal planes, precipitated from (a) $2.5 \mathrm{mM}$, and (b) $10 \mathrm{~mm}$ calcium chloride solutions. Top-down and side-views of the STORM images showing the clustered QDs, superimposed on the form of the crystal as determined by WFM. The STORM data are displayed in a colour map ranging from blue to red according to the detected number of photons per localisation. The corresponding histograms showing the localisation precision in the $\mathrm{x}-\mathrm{y}$ plane (lateral) and $\mathrm{z}$ depth component, and the number of photons per localisation are given in (c) and (d). Scale bars are $1 \mu \mathrm{m}$. 


\section{STORM}

a
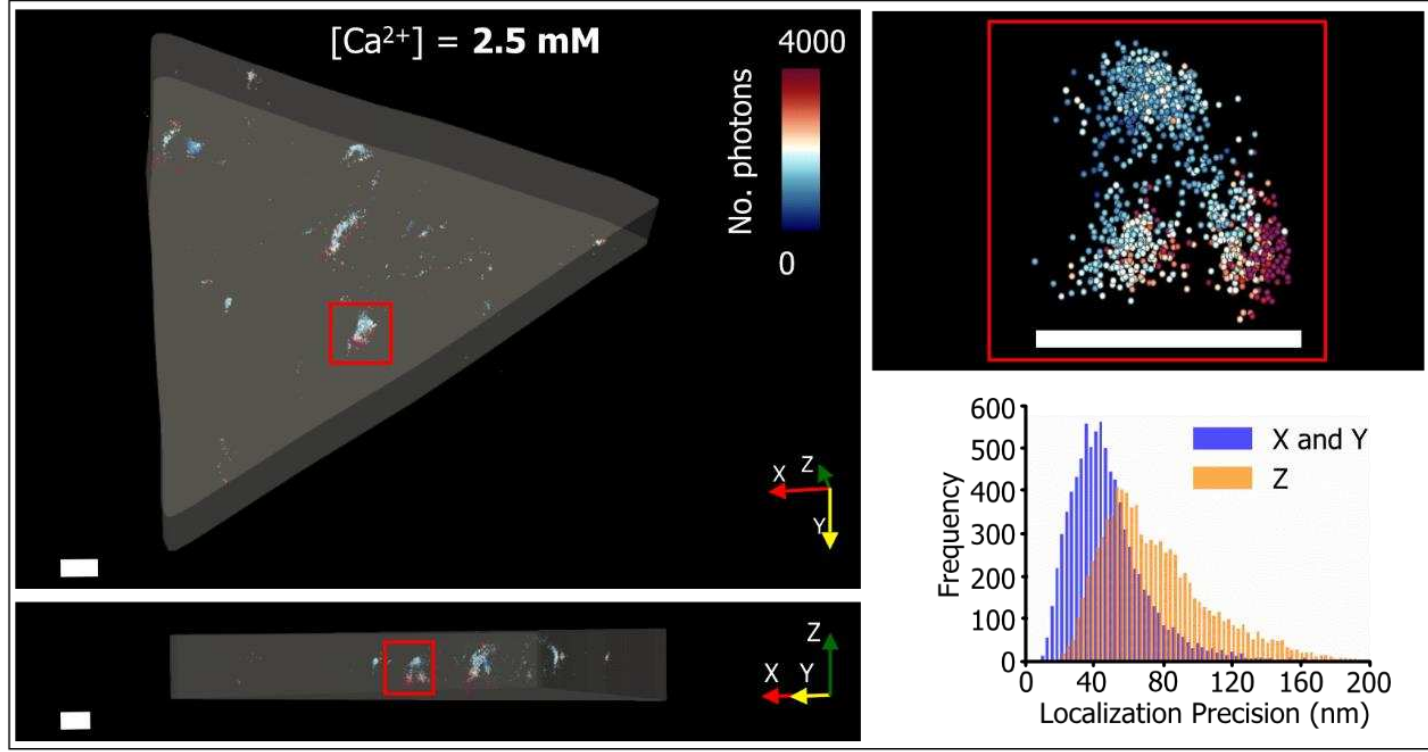

b
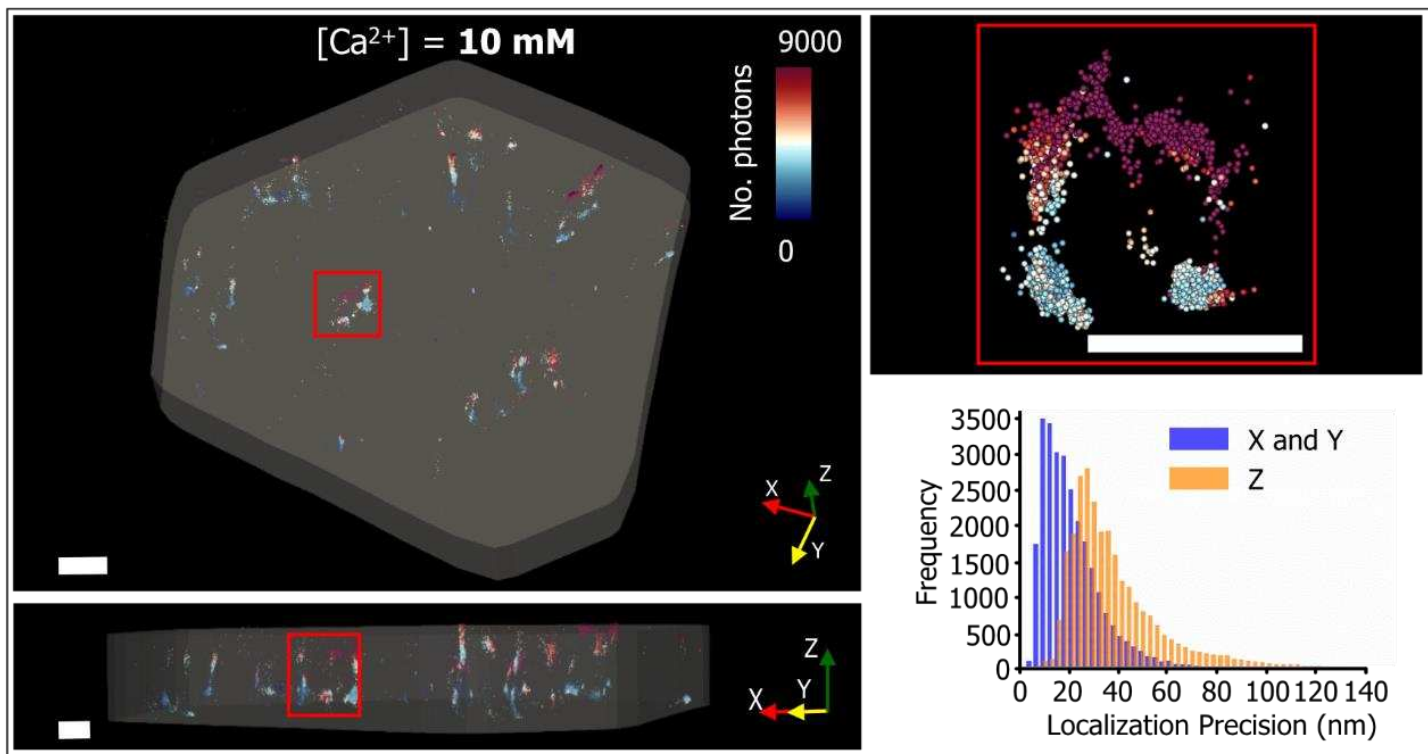

\section{BCDI}

C

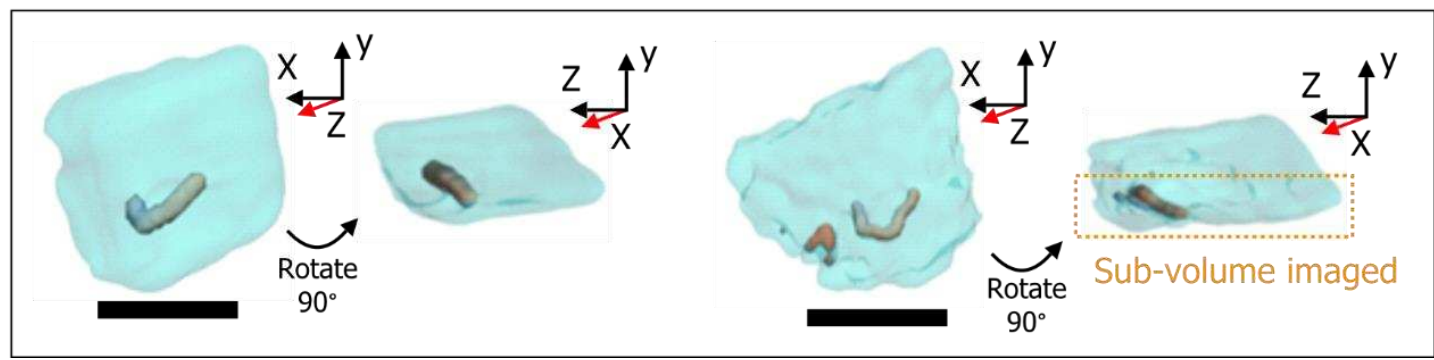

Figure 3. Dislocation Loops in Oriented Calcite Crystals as Observed by Stochastic Optical

Reconstruction Microscopy and Bragg Coherent Diffraction Imaging. (a) and (b) 3D STORM image reconstructions of $\{012\}$ oriented calcite nanocomposite crystals precipitated from (a) $2.5 \mathrm{mM}$ and (b) $10 \mathrm{mM}$ calcium chloride solutions. Top-down and side-view volume 
renderings of sub-sections of the crystals are shown and the STORM reconstructions are superimposed on the forms of the crystals as determined by WFM. Selected area magnifications (green boxes) highlight some of the dislocation loops, and the corresponding histograms are given. STORM data are displayed in a colour map ranging from blue to red according to the detected number of photons per localisation. (c) BCDI image reconstruction of 2 calcite crystals with $\{012\}$ basal planes, where each exhibits a single dislocation loop. Their location within the crystal, is indicated by the semi-transparent render of the crystals electron density projection. Top-down, and corresponding side views of two crystals are presented. Panel (c) is adapted from Ihli et al. ${ }^{[18]}$ 


\section{Graphical Abstract}

This work presents a novel, non-destructive strategy for visualizing dislocations in large synthetic crystals. Using single crystals of calcite containing quantum dots as a model system it is demonstrated that the nanoparticles form an atmosphere around the dislocations, enabling their visualisation using three-dimensional stochastic optical reconstruction microscopy (STORM).

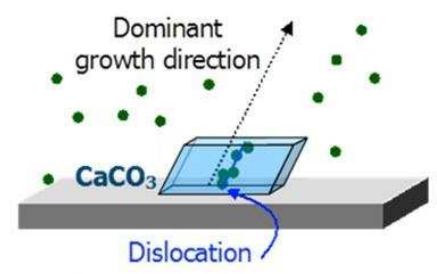

Crystals containing nanoparticles

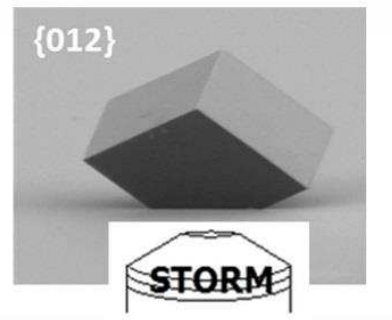

Super resolution microscopy

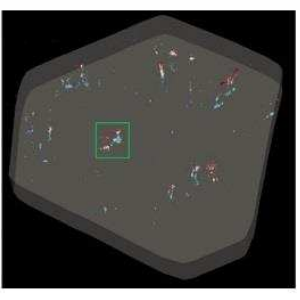

Image Dislocations

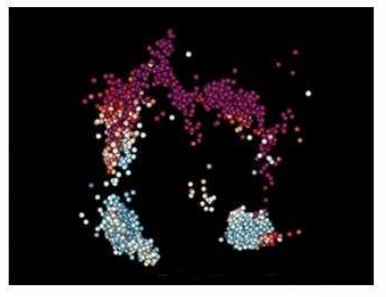

Multiple dislocation loops 